\section{Integral HOSM Semiglobal Controller for Finite-Time Exact Compensation of Unmatched Perturbations}

\author{
Antonio Estrada and Leonid M. Fridman
}

\begin{abstract}
The present technical note studies a class of nonlinear systems with unmatched perturbations. The combination of integral high-order sliding modes with the hierarchical quasi-continuous controller is proposed allowing finite-time exact compensation of unmatched perturbations with semiglobal convergence features for both regulation and tracking.
\end{abstract}

Index Terms-High-order sliding modes (HOSM), nonlinear block controllable form (NBC-form), sliding mode (SM) control.

\section{INTRODUCTION}

It is a known issue that classical sliding mode (SM) control [22] is not able to compensate unmatched perturbations [25].

Combinations of different robust techniques and SM have been applied to deal with systems with unmatched uncertainties [11]-[14]. In order to reduce the effects of the unmatched uncertainties [9] proposes a method that combines $\mathcal{H}_{\infty}$ and integral sliding mode control. The main idea is to choose a projection matrix, which ensures that unmatched perturbations are not amplified and moreover minimized. For uncertain nonlinear systems in strict-feedback form [19], [20] develop the technique known as backstepping where a virtual control based on Lyapunov methods is constructed step by step. In a similar manner to backstepping, Multiple surface sliding control is proposed in [21] to simplify the controller design of systems where model differentiation is difficult.

The combination of the backstepping design and sliding mode control is studied in [6] for systems in strict-feedback form with parameter uncertainties and extended to the multi-input case in [7]. The procedure proposed in [6], [7] reduces the computational load, as compared with the standard backstepping strategy, because it only retains $n-2$ steps of the original backstepping technique, coupling them with with an auxiliary second order subsystem to which a second order sliding mode control is applied. In [8] the combination of dynamical adaptive backstepping and first and second order sliding mode control is applied to both triangular and nontriangular uncertain observable minimum phase nonlinear systems.

Another approach to the problem of unmatched uncertainty compensation is based on the nonlinear block controllable form (NBC-form) [1]. In [1] the sliding mode technique is applied to compensate the matched perturbations. A high gain approach is used to achieve compensation of unmatched uncertainties and stabilization of the sliding mode dynamics. In [15] a sliding mode controller is designed using the combination of block control [16], a sigmoid aproximation to the integral sliding mode control [17] and nested sliding mode control [18]. A

Manuscript received June 01, 2009; revised November 12, 2009 and April 29, 2010; accepted July 22, 2010. Date of publication August 16, 2010; date of current version November 03, 2010. This work was supported by the Mexican CONACyT (Consejo Nacional de Ciencia y Tecnologia), CVU 211269 Grant 56819. Recommended by Associate Editor X. Chen.

The authors are with Department of Control and Robotics, Engineering Faculty, Universidad Nacional Autónoma de México (UNAM), Mexico City C.P. 04510, México (e-mail: xheper@yahoo.com; lfridman@servidor.unam.mx).

Color versions of one or more of the figures in this technical note are available online at http://ieeexplore.ieee.org.

Digital Object Identifier 10.1109/TAC.2010.2067270 coordinate transformation is applied to design a nonlinear sliding manifold. This transformation requires smoothness of each virtual control, that is why sigmoid, instead of signum functions are used. With the use of the high gain approach in [1] and sigmoid functions in [15], [18] they prove that asymptotic tracking is achieved.

In [2] a new design algorithm for systems in strict-feedback form, a special case of the BC-form, is proposed. This algorithm achieves finite-time exact tracking of the desired output in the presence of smooth unmatched perturbations. These features are accomplished via the usage of quasi-continuous high-order sliding modes (HOSM) and a hierarchical design approach. In the first step the desired dynamic for the first state is defined by the desired tracking signal. After the first step the desired dynamic for each state is defined by the previous one. Each virtual control is divided into two parts, the first one is intended to compensate the nominal nonlinear part of the system and the second one is aimed to achieve the desired dynamics in spite of perturbations.

Difficulties arise when initial conditions lead to big initial errors because then the smoothness needed to achieve and keep the HOSM of each virtual control could be broken in some of them leading to loss of control. One possible solution is to increase the gains of the HOSM term included in each virtual control, nevertheless it goes against a key motivation of the algorithm which is to reduce discontinuous control gain via the use of information on the known nominal part of the system [24]. The solution proposed in the present technical note is to apply the integral HOSM approach reported in [3] in which the desired reference is reached by means of a previously designed auxiliary smooth trajectory that depends on the initial conditions of the error and its derivatives up to the order of the HOSM control used. Thus each state starts in the proper auxiliary sliding motion and the entire internal dynamics remain controlled from the beginning.

The present technical note proceeds as follows. In Section II the class of nonlinear systems to be treated and the problem formulation are described. Section III introduces the Quasi-continuous control [4], the hierarchical design algorithm proposed in [2] and finally introduces the integral HOSM [3]. Section IV presents the modification to the hierarchical design algorithm and its convergence proof. In Section V the algorithm is applied and simulation results are presented. The note then concludes with a brief comment on the proposed algorithm.

\section{PRoBlem STATEMENT}

Consider a class of nonlinear systems presented in the special NBCform [1]

$$
\left.\begin{array}{l}
\dot{x}_{1}=f_{1}\left(x_{1}, t\right)+B_{1}\left(x_{1}, t\right) x_{2}+\omega_{1}\left(x_{1}, t\right) \\
\dot{x}_{i}=f_{i}\left(\bar{x}_{i}, t\right)+B_{i}\left(\bar{x}_{i}, t\right) x_{i+1}+\omega_{i}\left(\bar{x}_{i}, t\right) \\
\dot{x}_{n}=f_{n}(x, t)+B_{n}(x, t) u+\omega_{n}(x, t) \\
i=2, \ldots, n-1
\end{array}\right\}
$$

where $x \in R^{n}$ is the state vector, $x_{i} \in R, \bar{x}_{i}=\left[x_{1} \ldots x_{i}\right]^{T} ; u \in R$ is the control vector. Moreover $f_{i}\left(\bar{x}_{i}, t\right)$ and $B_{i}\left(\bar{x}_{i}, t\right)$ are smooth functions, $\omega_{i}$ is a bounded unknown perturbation term due to parameter variations and external disturbances with at least $n-i$ bounded derivatives w.r.t. system (1). $B_{i} \neq 0$ for $x \in X \subset R^{n}, t \in[0, \infty)$.

The relative degree, $\varrho_{d}$, of the output is assumed to be constant and known. To simplify the exposition, only the case of a $\rho_{d}=n$ is considered i.e. $y=x_{1}$; however, if zero dynamics stability is assumed, the approach can be used in the case of $\rho_{d}<n$. The whole state is assumed to be known. The goal is to achieve exact tracking of a smooth desired signal, $y_{d}$ by the output $y$.

In [2], HOSM and feedback compensation is introduced through several virtual controls in order to ensure the desired tracking. The results 
achieved in [2] are local and, for the sake of completeness, described in next section.

\section{HierarchicAl QuASI-Continuous Controller DESIGN [2]}

In the first part of this section the Quasi-continuous controller is briefly introduced, after that the design algorithm reported in [2] is restated for reader convenience and in the third part the integral HOSM approach is introduced.

\section{A. Quasi-Continuous Controller [4]}

Consider a single-input-single-output system of the form

$$
\begin{aligned}
\dot{\xi} & =a(t, \xi)+b(t, \xi) u, \quad \xi \in R^{n}, \quad u \in R \\
\sigma:(t, \xi) & \mapsto \sigma(t, \xi) \in R
\end{aligned}
$$

where $\sigma$ is the measured output of the system, $u$ is the control. Smooth functions $a, b, \sigma$ are assumed to be unknown, the dimension $n$ can also be uncertain. The task is to achieve $\sigma \equiv 0$.

It is assumed that system (2) has a constant and known relative degree $r$. From [23] it follows that $\sigma^{(r)}=h(t, \xi)+g(t, \xi) u, g(t, \xi) \neq 0$ holds, where $h(t, \xi)=\left.\sigma^{(r)}\right|_{u=0}, g(t, \xi)=(\partial / \partial u) \sigma^{(r)}$. If the inequalities $0<K_{m} \leq(\partial / \partial u) \sigma^{(r)} \leq K_{M},\left|\sigma^{(r)}\right|_{u=0} \mid \leq C$ are fulfilled for some $K_{m}, K_{M}, C>0$. Trajectories of (2) are assumed infinitely extendible in time for any Lebesgue-measurable bounded control $\mathrm{u}(\mathrm{t}, \mathrm{x})$. The next differential inclusion is implied

$$
\sigma^{(r)} \in[-C, C]+\left[K_{m}, K_{M}\right] u .
$$

The above problem can be solved by the Quasi-continuous controller [4], that is constructed such that $\sigma=\dot{\sigma}=\ldots=\sigma^{(r-1)}=0$ is established in finite time

$$
\begin{aligned}
u & =-\alpha \Psi_{r-1, r}\left(\sigma, \dot{\sigma}, \ldots, \sigma^{(r-1)}\right) \\
\varphi_{0, r} & =\sigma, \quad N_{0, r}=|\sigma|, \quad \Psi_{0, r}=\varphi_{0, r} / N_{0, r}=\operatorname{sign} \sigma \\
\varphi_{i, r} & =\sigma^{(i)}+\beta_{i} N_{i-1, r}^{(r-i) /(r-i+1)} \Psi_{i-1, r} \\
N_{i, r} & =\left|\sigma^{(i)}\right|+\beta_{i} N_{i-1, r}^{(r-i) /(r-i+1)} \\
\Psi_{i, r}(\cdot) & =\varphi_{i, r} / N_{i, r} ; \quad i=0, \ldots, r-1
\end{aligned}
$$

where $\beta_{1}, \ldots, \beta_{r-1}$, are positive numbers.

Theorem 1: [4] Provided that $\beta_{1}, \ldots, \beta_{r-1}, \alpha>0$ are chosen sufficiently large in the listed order, the above design results in the r-sliding homogeneous controller

$$
u=-\alpha \Psi_{r-1, r}\left(\sigma, \dot{\sigma}, \ldots, \sigma^{(r-1)}\right)
$$

providing for the finite-time stability of (3), (6). The finite-time stable r-sliding mode $\sigma \equiv 0$ is established in system (2), (6).

\section{B. Hierarchical Quasi-Continuous Controller Design Algorithm [2]}

Each state is seen as a function of the previous states, $x_{i+1}(t)=$ $\phi_{i}\left(\bar{x}_{i}(t)\right)$, i.e., as a virtual control. For example consider the state $x_{2}$ in (1) as a virtual control, then uncertainty $\omega_{1}\left(x_{1}, t\right)$ is seen as matched. The next algorithm is proposed to achieve tracking of $y_{d}$.

Step 1: Defining $x_{2}=\phi_{1}$, the $n$-sliding homogeneous quasi-continuous controller is included in $\phi_{1}$, where $\phi_{1}$ is an $n-1$ times differentiable function defined as

$$
\begin{aligned}
\phi_{1}\left(x_{1}, t, u_{1,1}\right) & =B_{1}\left(x_{1}, t\right)^{-1}\left\{-f_{1}\left(x_{1}, t\right)+u_{1,1}\right\} \\
\dot{u}_{1,1} & =u_{1,2} \\
& \vdots \\
\dot{u}_{1, n-1} & =-\alpha_{1} \Psi_{n-1, n}\left(\sigma_{1}, \dot{\sigma}_{1}, \ldots, \sigma_{1}^{(n-1)}\right)
\end{aligned}
$$

where $\sigma_{1}=x_{1}-y_{d}$. The first part of control $\phi_{1}\left(x_{1}\right)$ is aimed at compensating the nominal part of the system. The second part, $u_{1,1}$, is aimed at compensating perturbations; it is introduced through $n-1$ integrators in order to maintain the relative degree of $x_{1} . \Psi_{n-1, n}(\cdot)$ is defined as in (5) with the substitutions $r=n, \sigma=\sigma_{1}$.

The derivatives $\sigma_{1}, \dot{\sigma}_{1}, \ldots, \sigma_{1}^{(n-1)}$ are calculated by means of robust differentiators with finite-time convergence [5].

The convergence proof is based on the assumption of a constant and known relative degree, equal to $n$, of the output which leads to the existence of a differential inclusion $\sigma_{1}^{n} \in\left[-C_{1}, C_{1}\right]+\left[K_{m 1}, K_{M 1}\right] \dot{u}_{1, n-1}$ for some constants $C_{1}, K_{m 1}, K_{M 1}$ and thus the establishment of the finite-time stable $n$-sliding mode for the constrain $\sigma_{1}$. An analog procedure is applied for each state. As it was mentioned before only local convergence can be assured.

Step $i$ : The desired dynamics for $x_{i}$ is $\phi_{i-1}\left(x_{i}\right)$. The control proposed is analogous to (7), but with some changes in the order

$$
\begin{aligned}
\phi_{i}\left(\bar{x}_{i}, t, u_{i, 1}\right) & =B_{i}\left(\bar{x}_{i}, t\right)^{-1}\left\{-f_{i}\left(\bar{x}_{i}, t\right)+u_{i, 1}\right\} \\
\dot{u}_{i, 1} & =u_{i, 2} \\
& \vdots \\
\dot{u}_{i, n-i} & =-\alpha_{i} \Psi_{n-i, n-i+1}\left(\sigma_{i}, \dot{\sigma}_{i}, \ldots, \sigma_{i}^{(n-i)}\right)
\end{aligned}
$$

where $\sigma_{i}=x_{i}-\phi_{i-1}$ and $\Psi_{n-i, n-i+1}(\cdot)$ is defined as in (5), with the proper substitution $\sigma=\sigma_{i}$ in those equations.

Step n: In this step the real control is calculated, $\sigma_{n}=x_{n}-\phi_{n-1}$ then

$$
\begin{aligned}
u & =B_{n}(x, t)^{-1}\left\{-f_{n}(x, t)+u_{n, 1}\right\} \\
\text { where } \quad u_{n, 1} & =-\alpha_{n} \operatorname{sign}\left(\sigma_{n}\right) .
\end{aligned}
$$

Remark 1: If the existence of perturbations and desired signal derivatives of the proper order can be assured, the order of the HOSM in each virtual control could be increased and thus the real control can be softened.

Theorem 2: [2] Provided that $\omega_{i}$ in system (1) and $y_{d}$ are smooth functions with $n-i$ and $n$ bounded derivatives respectively the above hierarchic design results in an ultimate controller $u=\phi_{n}\left(x_{n}\right)$ providing for the finite time stability of $\sigma_{1}=x_{1}-y_{d}=\dot{\sigma}_{1}=\ldots=$ $\sigma_{1}^{(n-1)}=0$ in system (1).

Remark 2: The above theorem is valid only locally and stability is strongly dependent on the initial conditions of the system.

\section{Integral HOSM [3]}

In order to illustrate the convenience of the integral HOSM approach in combination with the above described design algorithm, consider the state $x_{n}$ and recall the first step of the convergence proof for the control (9) as stated in [2]:

- For the state $n$

$$
\begin{aligned}
\dot{x}_{n} & =f_{n}+B_{n} u+\omega_{n}(x, t) \\
\text { with } \quad u & =B_{n}^{-1}\left\{-f_{n}+\alpha_{n} \operatorname{sign}\left(\sigma_{n}\right)\right\} \\
\sigma_{n} & =x_{n}-\phi_{n-1} ; \quad \phi_{n-1} \quad \text { sufficiently smooth. }
\end{aligned}
$$

Then $\dot{\sigma}_{n}=-\alpha_{n} \operatorname{sign}\left(\sigma_{n}\right)+\omega_{n}(x, t)+\dot{\phi}_{n-1}$ and taking $\alpha_{n} \geq$ $\left|\omega_{n}\right|+\left|\dot{\phi}_{n-1}\right|$ provides for the appearance of a 1-sliding mode for the constraint $\sigma_{n}$ after a finite time $T n$. Thus the subsystem

$$
\dot{x}_{n-1}=f_{n-1}+B_{n-1} x_{n-1}+\omega_{n-1}\left(\bar{x}_{n-1}, t\right)
$$

could be unstable in the transient, when $x_{n} \neq \phi_{n-1}$. The same can be said, unless the system is bounded-input-bounded-state (BIBS), for each of the remaining states used as virtual controls before they reach the desired dynamics. 
In order to overcome the problem of transient dynamics, use of integral High-order sliding modes is proposed. The main idea is that each virtual control starts in the sliding mode of the proper order from the very beginning. The procedure is as follows.

Consider the r-sliding controller (6) and suppose a transient trajectory $\sigma(t, x(t))=\rho(t), t_{0} \leq t \leq t_{f}$ such that

$$
\left.\begin{array}{l}
\rho\left(t_{0}\right)=\sigma\left(t_{0}\right), \ldots, \rho^{(j)}\left(t_{0}\right)=\sigma^{(j)}\left(t_{0}\right) \\
j=1, \ldots, r-1 ; \quad \rho(t) \equiv 0 \forall t \geq t_{f}
\end{array}\right\}
$$

Integral r-Sliding Mode: Let $\rho^{(r-1)}(t)$ be a Lipschitz function, then it has a globally bounded derivative $\rho^{(r)}(t)$ almost everywhere, and the new output $\Sigma(t, x)=\sigma(t, x)-\rho(t)$ satisfies $0<K_{m} \leq(\partial / \partial u) \Sigma^{(r)} \leq K_{M},\left|\Sigma^{(r)}\right|_{u=0} \mid \leq C$ with some changed constants $K_{m}, K_{M}, C>0$.

Let the $(r-1)$ - smooth function $\rho(t)$ satisfying (10) have the form

$$
\rho=\left(t-t_{f}\right)^{r}\left(c_{0}+c_{1}\left(t-t_{0}\right)+\ldots+c_{r-1}\left(t-t_{0}\right)^{r-1}\right) .
$$

Parameters $c_{i}$ are now to be found from conditions (10) after $t_{f}$ is assigned. In order to avoid the necessity of very large control values to reach the $r$-sliding mode $\vec{\sigma}\left(t_{0}\right)=0$ (i.e. $\sigma=\dot{\sigma}=\ldots=\sigma^{(r-1)}=0$ ) due to far distanced initial values, or a very low convergence rate if $\vec{\sigma}\left(t_{0}\right)$ is close to zero, instead of a constant let $t_{f}-t_{0}$ be a continuous positive-definite r-sliding homogeneous function of the initial conditions $\vec{\sigma}\left(t_{0}\right)$ of homogeneity degree 1 , i.e.

$$
t_{f}-t_{0}=T\left(\vec{\sigma}\left(t_{0}\right)\right), \quad T\left(d_{\kappa} \vec{\sigma}\right)=\kappa T(\vec{\sigma}) \quad \forall \kappa>0 .
$$

Theorem 3: [3] The function $\rho\left(t-t_{0}, \vec{\sigma}\left(t_{0}\right)\right)$ is uniquely determined by (10), (11), (12). Then with any sufficiently large $\alpha$, independent of the initial conditions $\vec{\sigma}\left(t_{0}\right)$, the controller (6):

$$
\begin{gathered}
u=\alpha \Psi_{r-1, r}\left(\Sigma, \dot{\Sigma}, \ldots, \Sigma^{(r-1)}\right) \\
\Sigma(t, x)=\left\{\begin{array}{cl}
\sigma(t, x) & t_{0} \leq t \leq t_{0}+T\left(\vec{\sigma}\left(t_{0}\right)\right) \\
-\rho\left(t-t_{0}, \vec{\sigma}\left(t_{0}\right)\right) & t \geq t_{0}+T\left(\vec{\sigma}\left(t_{0}\right)\right)
\end{array}\right.
\end{gathered}
$$

establishes the finite-time-stable r-sliding mode $\sigma \equiv 0$ with the transient time (12). The equality $\left.\sigma(t, x(t))=\rho\left(t-t_{0}, \vec{\sigma}\left(t_{0}\right)\right)\right)$ is kept during the transient process.

The function used in this technical note for $T\left(\vec{\sigma}\left(t_{0}\right)\right)$ is the one reported in [3], which expression is

$$
\begin{gathered}
T\left(\vec{\sigma}\left(t_{0}\right)\right)=\lambda\left(\left|\sigma\left(t_{0}\right)\right|^{p / r}+\left|\dot{\sigma}\left(t_{0}\right)\right|^{p /(r-1)}+\right. \\
\left.\ldots+\left|\sigma^{(r-1)}\left(t_{0}\right)\right|^{p}\right)^{1 / p} ; \quad p, \lambda>0 .
\end{gathered}
$$

As previously mentioned the initial conditions independence feature achieved by the integral HOSM will be used in the Hierarchical Quasicontinuous design; the details are explained in the next section.

\section{INTEGRAL HIERARCHICAL QUASI-CONTINUOUS CONTROLLER}

\section{A. Hierachical Quasi-Continuous Controller Modification}

The only modification to the above described hierachical quasi-continuous controller is the substitution of each $\sigma_{i}$ for $\Sigma_{i}=\sigma_{i}-\rho_{i}$, as described next.
Step $i$ : The $i$-th sliding surface is chosen as $\Sigma_{i}=\sigma_{i}-\rho_{i}$ where $\sigma_{i}=x_{i}-\phi_{i-1}$ (with the exception $\sigma_{1}=x_{1}-y_{d}$ )

$$
\begin{aligned}
\phi_{i}\left(\bar{x}_{i}, t, u_{i, 1}\right)= & B_{i}\left(\bar{x}_{i}, t\right)^{-1}\left\{-f_{i}\left(\bar{x}_{i}, t\right)+u_{i, 1}\right\} \\
\dot{u}_{i, 1}= & u_{i, 2} \\
& \vdots \\
\dot{u}_{i, n-i}= & -\alpha_{i} \Psi_{n-i, n-i+1} \\
& \times\left(\Sigma_{i}, \dot{\Sigma}_{i}, \ldots, \Sigma_{i}^{(n-i)}\right) .
\end{aligned}
$$

$\Psi_{n-i, n-i+1}$ is defined as in (5), obviously using $\sigma=\Sigma_{i}$ in those equations and where $\rho_{i}$ fulfills condition (10):

$$
\left.\begin{array}{c}
\rho_{i}\left(t_{0}\right)=\sigma\left(t_{0}\right), \ldots, \rho_{i}^{(n-i)}\left(t_{0}\right)=\sigma_{i}^{(n-i)}\left(t_{0}\right) \\
\rho_{i}(t) \equiv 0 \quad \forall t \geq t_{f i}
\end{array}\right\}
$$

and is constructed according to (11) setting $r=n-i+1$

$$
\begin{array}{r}
T\left(\overrightarrow{\sigma_{i}}\left(t_{0}\right)\right)=\lambda_{i}\left(\left|\sigma_{i}\left(t_{0}\right)\right|^{p / n-i+1}+\left|\dot{\sigma}_{i}\left(t_{0}\right)\right|^{p /(n-i)}+\right. \\
\left.\cdots+\left|\sigma_{i}^{(n-i)}\left(t_{0}\right)\right|^{p}\right)^{1 / p}
\end{array}
$$

Step $n: \Sigma_{n}=\sigma_{n}-\rho_{n}$ where $\sigma_{n}=x_{n}-\phi_{n-1}$

$$
\begin{aligned}
u & =B_{n}(x, t)^{-1}\left\{-f_{n}(x, t)+u_{n, 1}\right\} \\
\text { where } u_{n, 1} & =-\alpha_{n} \operatorname{sign}\left(\Sigma_{n}\right) .
\end{aligned}
$$

Theorem 4: If system (1) is BIBS then provided that $\omega_{i}$ and $y_{d}$ are smooth functions with $n-i$ and $n$ bounded derivatives respectively the above hierarchic design results in the controller (18) that assures the finite time stability of $\sigma_{1}=x_{1}-y_{d}=\dot{\sigma}_{1}=\ldots=\sigma_{1}^{(n-1)}=0$ in system (1) independently of their initial conditions $x\left(t_{0}\right)$.

Remark 3: Observe that the BIBS condition is only a sufficient but not necessary condition as it can be seen in the convergence proof.

\section{B. Convergence Proof}

- Consider the state $x_{n}$

$$
\begin{aligned}
\dot{x}_{n}= & f_{n}+B_{n} u+\omega_{n}(x, t) \\
\text { with } \quad u= & B_{n}^{-1}\left\{-f_{n}+\alpha_{n} \operatorname{sign}\left(\Sigma_{n}\right)\right\} \\
\Sigma_{n}= & \sigma_{n}-\rho_{n}, \quad \sigma_{n}=x_{n}-\phi_{n-1}, \quad \phi_{n-1} \\
& \text { sufficiently smooth. }
\end{aligned}
$$

Then $\dot{\Sigma}_{n}=-\alpha_{n} \operatorname{sign}\left(\sigma_{n}\right)+\omega_{n}(x, t)+\dot{\phi}_{n-1}-\dot{\rho}_{n}$ and taking $\alpha_{n} \geq\left|\omega_{n}\right|+\left|\dot{\phi}_{n-1}\right|+\left|\dot{\rho}_{n}\right|$ provides for the appearance of a 1 -sliding mode for the constraint $\Sigma_{n}$ after $t=t_{0}$, i.e. since the beginning, and for $\sigma_{n}$ after $T_{n}=t_{f n}-t_{0}=\lambda_{n}\left(\left|\sigma_{n}\left(t_{0}\right)\right|\right)$ choosing $p=1$ for (17).

- Now for the state $x_{n-1}$, with $\phi_{n-1}$ defined according to (15) and $\Sigma_{n-1}=\sigma_{n-1}-\rho_{n-1}, \sigma_{n-1}=x_{n-1}-\phi_{n-2}$ then

$$
\begin{aligned}
\dot{\Sigma}_{n-1} & =\dot{x}_{n-1}-\dot{\phi}_{n-2}-\dot{\rho}_{n-1} \\
& =f_{n-1}+B_{n-1} x_{n}+\omega_{n-1}-\dot{\rho}_{n-1}-\dot{\phi}_{n-2} \\
& =f_{n-1}+B_{n-1}\left(\phi_{n-1}+\rho_{n-1}\right)+\omega_{n-1}-\dot{\rho}_{n-1}-\dot{\phi}_{n-2} .
\end{aligned}
$$

The function $f_{n-1}$, may not be compensated right from $t=t_{0}$ because of the arbitrary initial condition of $x_{n}$. However due to the 
BIBS condition $x_{n-1}$ remains bounded and after $t=t_{0}+T_{n}$, when $x_{n}=\phi_{n-1}$

$$
\begin{aligned}
& \dot{\Sigma}_{n-1}=u_{n-1,1}+\omega_{n-1}\left(\bar{x}_{n-1}, t\right)-\dot{\rho}_{n}-\dot{\phi}_{n-2} \\
& \ddot{\Sigma}_{n-1}=\dot{u}_{n-1,1}+\dot{\omega}_{n-1}\left(\bar{x}_{n-1}, t\right)-\ddot{\rho}_{n}-\ddot{\phi}_{n-2}
\end{aligned}
$$

that is (19) takes the form

$$
\begin{aligned}
\ddot{\Sigma}_{n-1} & =h_{n-1}(t, x)+g_{n-1}(t, x) u_{n-1} \\
\text { with } \quad h_{n-1}(t, x) & =\left.\ddot{\Sigma}_{n-1}\right|_{u_{n-1}=0} ; \\
g_{n-1}(t, x) & =\frac{\partial}{\partial u_{n-1}} \ddot{\Sigma}_{n-1} \\
u_{n-1} & =\dot{u}_{n-1,1} \\
& =-\alpha_{n-1} \Psi_{1,2}\left(\Sigma_{n-1}, \dot{\Sigma}_{n-1}\right) .
\end{aligned}
$$

If for some $K_{m_{n-1}}, K_{M_{n-1}}, C_{n-1}>0$ the inequalities $0<$ $K_{m_{n-1}} \leq\left(\partial / \partial u_{n-1}\right) \ddot{\Sigma}_{n-1} \leq K_{M_{n-1}},\left|\ddot{\Sigma}_{n-1}\right|_{u_{n-1}=0} \mid \leq C_{n-1}$ holds the next differential inclusion is implied

$$
\ddot{\Sigma}_{n-1} \in\left[-C_{n-1}, C_{n-1}\right]+\left[K_{m_{n-1}}, K_{M_{n-1}}\right] u_{n-1}
$$

and controller (21) keeps ( since it was established from $t_{0}$ ) stability of (22), (21). The finite-time stable 2-sliding mode is maintained for the constraint $\Sigma_{n-1}$ from $t_{0}$ and for $\sigma_{n-1}$ after $t_{f n-1}=t_{0}+T_{n}$.

The same procedure can be applied to each one of the states of (1).

Remark 4: As it was previously mentioned it becomes clear that the BIBS condition is not a necessary one, it will suffice that in each subsystem of (1)

$$
\dot{x}_{i}=f_{i}\left(\bar{x}_{i}, t\right)+B_{i}\left(\bar{x}_{i}, t\right) x_{i+1}+\omega_{i}\left(\bar{x}_{i}, t\right)
$$

$x_{i}$ remains bounded with the input $x_{i+1}$ bounded, at least during the time interval $t<t_{f i}$; because after that time $f_{i}$ is compensated. This BIBS condition may be relaxed with the application of the new advances in HOSM adjustment [10].

Notice that, with the use of integral HOSM in each virtual control, it is possible to introduce suitable dynamics on each of them. If direct application is used, in which only the input and the output is considered, this is not possible. The algorithm proposed in this technical note can be applied in physical systems such as synchronous machines and some underactuated manipulators that can be brought to the form (1).

\section{EXAMPLE}

Consider the perturbed third order system

$$
\begin{aligned}
& \dot{x}_{1}=2 \sin \left(x_{1}\right)+1.5 x_{2}+\omega_{1}\left(x_{1}, t\right) \\
& \dot{x}_{2}=0.8 x_{1} x_{2}+x_{3}+\omega_{2}\left(\bar{x}_{2}, t\right) \\
& \dot{x}_{3}=-1.5 x_{3}^{2}+2 u+\omega_{3}(x, t)
\end{aligned}
$$

where functions $\omega_{1}, \omega_{2}$ are the unmatched bounded perturbations and function $\omega_{3}$ is the matched perturbation. These functions were defined as follows:

$$
\begin{aligned}
\omega_{1}\left(x_{1}, t\right) & =0.2 \sin (t)+0.1 x_{1}+0.12 \\
\omega_{2}\left(\bar{x}_{2}, t\right) & =0.3 \sin (2 t)+0.2 x_{1}+0.2 x_{2}-0.4 \\
\omega_{3}(x, t) & =0.2 \sin (2 t)+0.2 x_{1}+0.3 x_{2}+0.2 x_{3}+0.3
\end{aligned}
$$

a controller that achieves tracking of $y_{d}=2 \sin (0.15 t)+4 \cos (0.1 t)-$ 4 by $x_{1}$ is desired. In addition to the previous perturbations the nominal
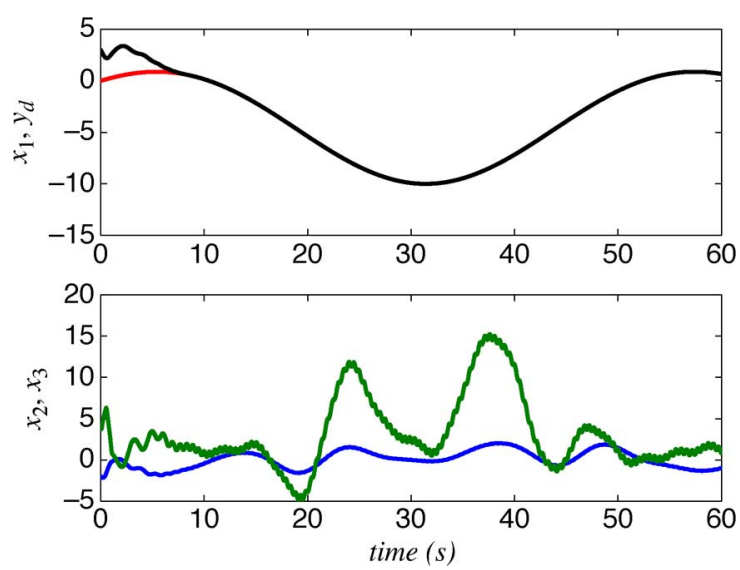

Fig. 1. States evolution $\left(x(0)=[3-24]^{T}\right)$.

compensation term of the first two virtual controls is not exact. The first sliding surface is $\Sigma_{1}=\sigma_{1}-\rho_{1}, \sigma_{1}=x_{1}-y_{d}$ and the virtual control for $x_{1}$

$$
\begin{aligned}
& \phi_{1}\left(x_{1}, t, u_{11}\right)=\frac{1}{1.5}\left\{-1.8 \sin \left(x_{1}\right)+u_{11}\right\} \\
& \dot{u}_{11}=u_{12} \\
& \dot{u}_{12}=-\alpha_{1} \Psi_{2,3}\left(\Sigma_{1}, \dot{\Sigma}_{1}, \ddot{\Sigma}_{1}\right) ; \\
& \Psi_{2,3}\left(\Sigma_{1}, \dot{\Sigma}_{1}, \ddot{\Sigma}_{1}\right) \\
& =\frac{\ddot{\Sigma}_{1}+2\left(\left|\dot{\Sigma}_{1}\right|+\left|\Sigma_{1}\right|^{2 / 3}\right)^{-1 / 2}\left(\dot{\Sigma}_{1}+\left|\Sigma_{1}\right|^{2 / 3} \operatorname{sign}\left(\Sigma_{1}\right)\right)}{\left|\ddot{\Sigma}_{1}\right|+2\left(\left|\dot{\Sigma}_{1}\right|+\left|\Sigma_{1}\right|^{2 / 3}\right)^{1 / 2}} \\
& \rho_{1}=\left(t-t_{f 1}\right)^{3}\left(c_{10}+c_{11}\left(t-t_{0}\right)+c_{12}\left(t-t_{0}\right)^{2}\right) \\
& T_{1}=\lambda_{1}\left(\left|\sigma_{1}\left(t_{0}\right)\right|^{2}+\left|\dot{\sigma}_{1}\left(t_{0}\right)\right|^{3}+\left|\ddot{\sigma}_{1}\left(t_{0}\right)\right|^{6}\right)^{1 / 6} .
\end{aligned}
$$

For the next state $\Sigma_{2}=\sigma_{2}-\rho_{2}, \sigma_{2}=x_{2}-\phi_{1}$ then

$$
\begin{aligned}
\phi_{2}\left(x_{2}, t, u_{21}\right) & =-0.7 x_{1} x_{2}+u_{21} \\
\dot{u}_{21} & =-\alpha_{2} \Psi_{1,2}\left(\Sigma_{2}, \dot{\Sigma}_{2}\right) \\
\Psi_{1,2}\left(\Sigma_{2}, \dot{\Sigma}_{2}\right) & =\frac{\dot{\Sigma}_{2}+\left|\Sigma_{2}\right|^{1 / 2} \operatorname{sign}\left(\Sigma_{2}\right)}{\left|\dot{\Sigma}_{2}\right|+\left|\Sigma_{2}\right|^{1 / 2}} \\
\rho_{2} & =\left(t-t_{f_{2}}\right)^{2}\left(c_{20}+c_{21}\left(t-t_{0}\right)\right) \\
T_{2} & =\lambda_{2}\left(\left|\sigma_{2}\left(t_{0}\right)\right|+\left|\dot{\sigma}_{2}\left(t_{0}\right)\right|^{2}\right)^{1 / 2} .
\end{aligned}
$$

Finally for state $x_{3}, \Sigma_{3}=\sigma_{3}-\rho_{3}, \sigma_{3}=x_{3}-\phi_{2}$

$$
\begin{aligned}
u & =\frac{1}{2}\left\{1.5 x_{3}^{2}+u_{31}\right\} \\
u_{31} & =-\alpha_{3} \operatorname{sign}\left(\Sigma_{3}\right) \\
\rho_{3} & =\left(t-t_{f_{3}}\right)\left(c_{30}\right) ; \quad T_{3}=\lambda_{3}\left(\left|\sigma_{3}\left(t_{0}\right)\right|\right) .
\end{aligned}
$$

Results obtained in simulations are shown in Figs. 1 and 2, using $\alpha_{1}=4, \alpha_{2}=10, \alpha_{3}=8, \lambda_{1}=6, \lambda_{2}=0.5, \lambda_{3}=1$. In Fig. 1 , $x(0)=\left[\begin{array}{lll}3 & -2 & 4\end{array}\right]^{T}$ is used. In Fig. $2, x(0)=\left[\begin{array}{lll}-3 & 1.5 & 2\end{array}\right]^{T}$ and a phase lead of $30 \mathrm{~s}$ is introduced in $y_{d}$ (seeFigs. 3 and 4 ).

\section{CONCLUSION}

The problem of control design for nonlinear systems with unmatched perturbations is treated in this technical note. A design algorithm providing finite-time exact tracking of a smooth desired signal is given. 


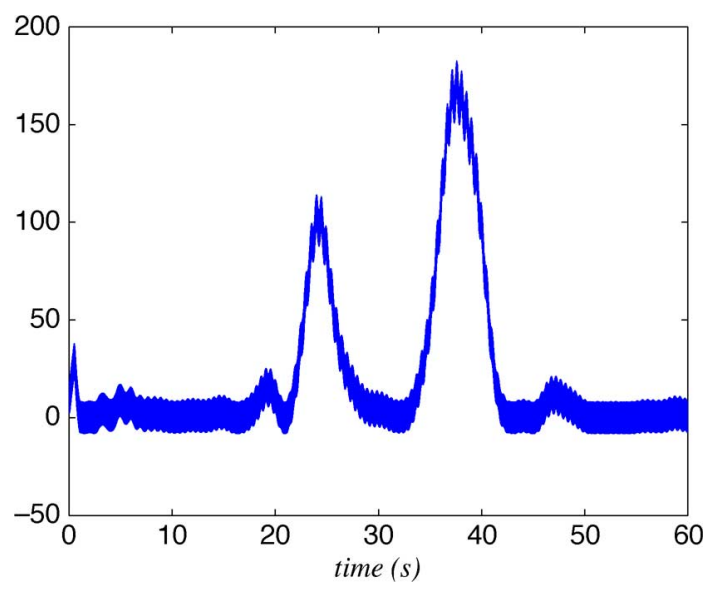

Fig. 2. Control signal $u$.
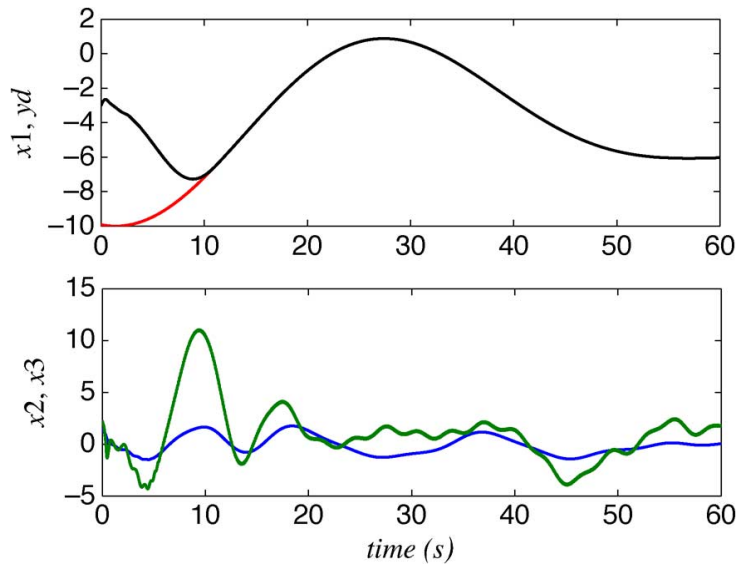

Fig. 3. States evolution $\left(x(0)=\left[\begin{array}{lll}-3 & 1.5 & 2\end{array}\right]^{T}\right)$.

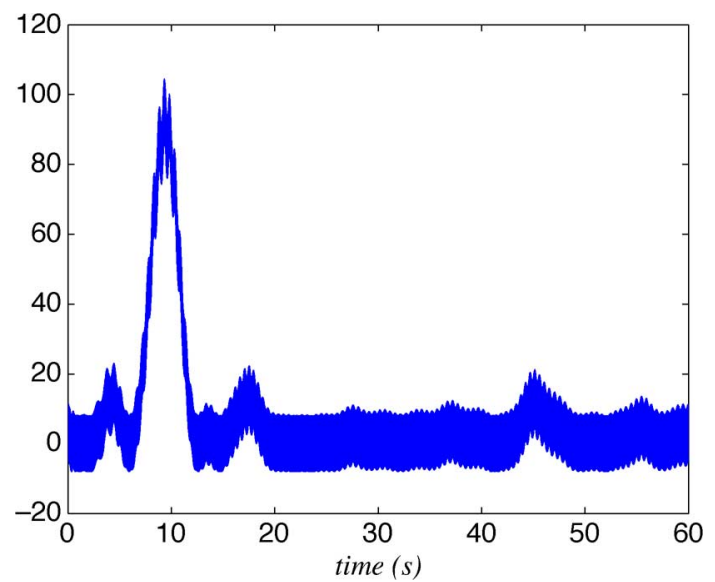

Fig. 4. Control signal $u$.

The proposed algorithm uses nested quasi-continuous HOSM in a hierarchical manner. The combination with integral HOSM in each virtual control allows introducing suitable dynamics on each of them thus providing semiglobal convergence.

\section{REFERENCES}

[1] A. G. Loukianov, "Robust block decomposition sliding mode control design," Math. Problems Eng., vol. 8, no. 4-5, pp. 349-365, 2002.
[2] A. Estrada and L. Fridman, "Exact compensation of unmatched perturbations via HOSM," in Proc. 47th IEEE Conf. Decision Control, Dec. 2008, pp. 278-282.

[3] A. Levant and L. Alelishvili, "Integral high-order sliding modes," IEEE Trans. Autom. Control, vol. 52, no. 7, pp. 1278-1282, Nov. 2007.

[4] A. Levant, "Quasi-continuous high-order sliding-mode controllers," IEEE Trans. Autom. Control, vol. 50, no. 11, pp. 1812-18162, Nov. 2005.

[5] A. Levant, "High-order sliding modes: Differentiation and output-feedback control," Int. J. Control, vol. 76, pp. 924-941, 2003.

[6] G. Bartolini, A. Ferrara, L. Giacomini, and E. Usai, "A combined backstepping/second order sliding mode approach to control a class of nonlinear systems," in Proc. IEEE Int. Workshop Variable Structure Syst. Tokyo, Japan, 1996, pp. 205-210.

[7] A. Ferrara and L. Giacomini, "On multi-input backstepping design with second order sliding modes for a class of uncertain nonlinear systems," Int. J. Control, vol. 71, no. 5, pp. 767-788, 1998.

[8] J. C. Scarratt, A. S. I. Zinober, R. E. Mills, M. Rios-Bolivar, A. Ferrara, and L. Giacomini, "Dynamical adaptive first and second-order sliding backstepping control of nonlinear nontriangular uncertain systems," ASME J. Dyn. Syst., Meas. Control, vol. 122, no. 4, pp. 746-752, 2000.

[9] F. Castaños and L. Fridman, "Analysis and design of integral sliding manifolds for systems with unmatched perturbations," IEEE Trans. Autom. Control, vol. 51, no. 5, pp. 853-858, May 2006.

[10] A. Levant and A. Michael, "Adjustment of high-order sliding-mode controllers," Int. J. Robust Nonlin. Control, vol. 15, pp. 1657-1672, 2009.

[11] R. Davis and S. K. Spurgeon, "A nonlinear control strategy for robust sliding mode performance in the presence of unmatched uncertainty," Int. J. Control, vol. 57, pp. 1107-1123, 1993.

[12] A. Poznyak, Y. B. Shtessel, and C. Jiménez, "Mini-max sliding mode control for multimodel linear time varying systems," IEEE Trans. Autom. Control, vol. 48, no. 12, pp. 2141-2150, Dec. 2003.

[13] H. H. Choi, "An LMI-based switching surface design method for a class of mismatched uncertain systems," IEEE Trans. Autom. Control, vol. 48, no. 9, pp. 1634-1638, Sep. 2003.

[14] W. Cao and J. Xu, "Nonlinear integral-type sliding surface for both matched and unmatched uncertain systems," IEEE Trans. Autom. Control, vol. 49, no. 8, pp. 1355-1360, Aug. 2004.

[15] H. Huerta-Avila, A. G. Loukianov, and J. M. Cañedoc, "Nested integral sliding modes of large sacale power systems," in Proc. 46thIEEE Conf. Decision Control, New Orleans, LA, Dec. 2007, pp. 1993-1998.

[16] A. G. Loukianov, "Nonlinear block control with sliding mode," Autom Remote Control, vol. 59, no. 7, pp. 916-933, 1998.

[17] V. I. Utkin, J. Guldner, and J. Shi, Sliding Mode Control in Electromechanical Systems. London, U.K.: Taylor \& Francis, 1999.

[18] A. Adhami-Mirhosseini and M. J. Yazdanpanah, "Robust tracking of perturbed systems by nested sliding mode control," in Proc. Int. Conf. Control Autom., Budapest, Hungary, 2005, pp. 44-48.

[19] R. A. Freeman and P. V. Kokotovic, Robust Nonlinear Control Design. Boston, MA: Birkhauser, 1996.

[20] M. Krstic, I. Kanellakopoulous, and P. Kokotovic, Nonlinear and Adaptive Control Design. New York: Wiley Interscience, 1995.

[21] M. C. Won and J. K. Hedrick, "Multiple surface sliding control of a class of uncertain nonlinear systems," Int. J. Control, vol. 64, no. 4, pp. 693-706, 1996.

[22] V. I. Utkin, Sliding Modes in Control and Optimization. Berlin, Germany: Springer Verlag, 1992.

[23] A. Isidori, Nonlinear Control Systems, 2nd ed. New York: SpringerVerlag, 1989.

[24] A. F. Filippov, Differential Equations With Discontinuous Right-Hand Side. Dordrecht, The Netherlands: Kluwer, 1988.

[25] B. Drazenovic, "The invariance conditions in variable structure systems," Automatica, vol. 5, no. 3, pp. 287-295, 1969. 